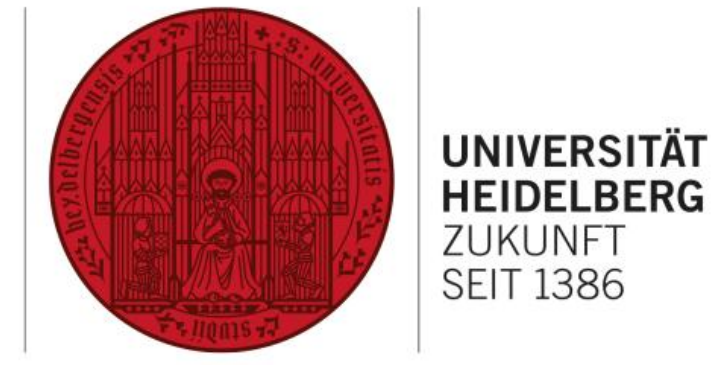

Zusammenfassung der Dissertation mit dem Titel

\title{
„Irrtum über normative Tatbestandsmerkmale. Eine Verortung der subjektiven Zurechnung innerhalb der verfassungsrechtlichen Koordinaten des Bestimmtheitsgrundsatzes und des Schuldprinzips ${ }^{66}$
}

Dissertation vorgelegt von Konstantina Papathanasiou

Erstgutachter: Prof. Dr. Gerhard Dannecker

Zweitgutachter: Prof. Dr. Jürgen Rath

Institut für deutsches, europäisches und internationales Strafrecht und Strafprozessrecht 


\begin{abstract}
Auf der Suche nach dem Vorsatzgegenstand fundiert die Autorin die strafrechtliche Irrtumslehre aus der Perspektive der Verfassung mit dem Ziel, die Schwächen der Parallelwertung in der Laiensphäre auszugleichen und den Bürger in verfassungskonformer Weise vor einer Ausuferung der strafrechtlichen Verantwortung zu schützen.
\end{abstract}

Die Autorin setzt sich einerseits mit den verschiedenen Ansätzen, die zur Lösung des Rätsels des Irrtums über normative Tatbestandsmerkmale entwickelt wurden ${ }^{1}$, andererseits mit der Trennung des Tatbestands- vom Verbotsirrtum und somit mit dem Gegensatzpaar von Vorsatz- und Schuldtheorie auseinander ${ }^{2}$. Eingehend wird insbesondere die Rückkehr $\mathrm{zu}$ der reichsgerichtlichen Rechtsprechung besprochen, die nicht schon dann vorliegt, wenn man die auf die rechtliche Wertung der Gesamttat bezogenen Irrtümer der Sphäre des Verbotsirrtums vorbehält, sondern erst dann, wenn man jede falsche rechtliche Bewertung aus dem Bereich des Tatbestandsirrtums herauszieht ${ }^{3}$. Die Ausführungen münden in zwei Desideraten: Erstens, »normativ geprägte Merkmale« im Allgemeinen zu thematisieren, so dass die anzuwendenden Vorsatzerfordernisse von vornherein bekannt sind und nicht von der willkürlichen Klassifizierung eines Merkmals abhängen; dabei sind individuelle Fähigkeiten des Täters schon für seinen Vorsatz mit zu berücksichtigen ${ }^{4}$. Zweitens, die Schuldtheorie einheitlich sowohl im Kern- als auch im Nebenstrafrecht anzuwenden ${ }^{5}$.

So kommt die Autorin zu ihrer Formel der »Widerspiegelung der gesetzgeberischen Grundentscheidung im Verständnishorizont des Täters" (abgekürzt: WGVT-Formel) ${ }^{6}$. Ausgehend von der Frage, ob das herkömmliche Verständnis von der sozialen Bedeutung der Norm, auf die die Parallelwertung in der Laiensphäre bezogen wird, den Anforderungen der Verfassung Rechnung trägt, wird nämlich die subjektive Zurechnung innerhalb der verfassungsrechtlichen Koordinaten des Bestimmtheitsgrundsatzes und des Schuldprinzips verortet. Im Einzelnen geht es bei diesem neuen Konzept um Folgendes:

I. Vor dem Hintergrund der Ergebnisse der ersten zwei Kapitel werden zuerst die Vor- und Nachteile des Kriteriums der Parallelwertung in der Laiensphäre erörtert. Daraus ergibt sich, dass ihr ursprünglicher Kern zwar richtig ist, sie selbst aber an verschiedenen Punkten krankt. An ihre Stelle soll ein neues Kriterium treten, das auf dem „Verständnishorizont des Täters" fußt ${ }^{7}$.

II. Anschließend wird der verfassungsrechtliche Ansatz aufgegriffen ${ }^{8}$ : Die fragwürdige und wenig aussagekräftige „soziale Bedeutung“ der Parallelwertung wird nun durch die auf die Verfassung bezogene WGVT-Formel ersetzt. In Betracht kommen insbesondere zwei wichtige Schutzschilde: der Bestimmtheitsgrundsatz und das Schuldprinzip. Bei der Analyse des ersten Schutzschildes ${ }^{9}$ wird über das Rechtsstaatprinzip gesprochen und sodann

\footnotetext{
${ }^{1}$ Siehe Erstes Kapitel.

2 Siehe Zweites Kapitel.

${ }^{3}$ Siehe Erstes Kapitel, insb. B. IX.

${ }^{4}$ Siehe Erstes Kapitel, D.

${ }^{5}$ Siehe Zweites Kapitel, C. und D.

${ }^{6}$ Siehe Drittes Kapitel. Zum Rahmenkonzept der Arbeit siehe bereits Papathanasiou, in: Festschrift für Claus Roxin, 2011, Bd. I, S. 467 ff.

${ }^{7}$ Siehe Drittes Kapitel, A.

${ }^{8}$ Siehe Drittes Kapitel, B.

${ }^{9}$ Siehe Drittes Kapitel, B. I.
} 
ausführlich die Bedeutung des Bestimmtheitsgrundsatzes dargestellt. Anschließend werden die Begriffe des „Normadressaten“ und der ,gesetzgeberischen Grundentscheidung“ präzisiert. Schließlich wird auf das Bedürfnis nach Rechtssicherheit eingegangen und zur Ergänzung wird die Problematik der Textontologie erwähnt. Bei der Analyse des zweiten Schutzschildes ${ }^{10}$ wird zuerst der Begriff der „Schuld“ erörtert und dabei die Bedeutung des Abstellens auf den konkreten Normadressaten aufgezeigt. Danach soll das Schuldprinzip gegen die Systemtheorie, die kein Individuum als selbstständig handelnde Einheit anerkennt, und vor allem gegen den neurobiologischen Determinismus, der die Willensfreiheit des Menschen ablehnt, abgesichert werden. Zur Ergänzung wird bei dieser Problematik der Unvollständigkeitssatz Gödels eingeführt.

III. Schließlich wird der Begriff der „Widerspiegelung“ erläutert ${ }^{11}$, d. h. wie sich die gesetzgeberische Grundentscheidung und der Verständnishorizont des Täters miteinander verbinden. Hier wird dem Kern der Lehre der Parallelwertung in der Laiensphäre uneingeschränkt gefolgt, dass nämlich für den Vorsatz bezüglich normativ geprägter Merkmale die Tatsachenkenntnis keineswegs ausreicht. Obwohl bisher niemand auf die Idee gekommen ist, sich hinsichtlich der Parallelwertung in der Laiensphäre mit der Bedeutung der „Parallelität“ zu beschäftigen, wird in diesem Teil der Arbeit die „Widerspiegelung“ philosophisch auf Grundlage der „transzendentalen Ästhetik“ Kants geklärt, allerdings ohne jegliche Letztbegründungsansprüche.

Im Rahmen der Formel der »Widerspiegelung der gesetzgeberischen Grundentscheidung im Verständnishorizont des Täters « wird somit der ursprüngliche Kern der herrschenden Lehre von der Parallelbewertung in der Laiensphäre tatsächlich bewahrt, zumal auf diesen durchaus korrekten Kern nicht verzichtet werden kann. Jedes Wollen setzt jedenfalls bereits Orientierung am Feststehenden voraus, es muss nämlich immer um eine Veränderung bei Beharrendem gehen, nicht um Veränderung schlechthin ${ }^{12}$.

Die WGVT-Formel minimiert nicht nur die vorhandenen theoretischen Nachteile. Die WGVT-Formel beweist Konsequenz mit und Treue zu ihren Prinzipien und gewährleistet daher Einheit innerhalb der Strafrechtsordnung und somit Rechtssicherheit. Sie gesteht ferner dem vorsatzausschließenden Tatbestandsirrtum genügend Raum zu, d. h. dem Täter steht bereits die Anwendung von $\S 16$ StGB in größtmöglichem Umfang zur Verfügung. Letzteres bedeutet wiederum ein Maximum an individueller Gerechtigkeit. ${ }^{13}$

\footnotetext{
${ }^{10}$ Siehe Drittes Kapitel, B. II.

${ }^{11}$ Siehe Drittes Kapitel, C.

${ }^{12}$ So das die Arbeit eröffnende Zitat von Emge, Sicherheit und Gerechtigkeit, S. 9.

${ }^{13}$ Siehe Zusammenfassung in fine.
} 